\title{
HELIX: A data-driven characterization of Brazilian land snails
}

\author{
Marcelo N. Almeida ${ }^{1}$, Rodolfo Alves de Oliveira ${ }^{1}$, Luiz Olmes ${ }^{2}$ \\ Gustavo S. Semaan ${ }^{1}$, Daniel de Oliveira ${ }^{3}$, Lúcio Santos ${ }^{4}$, and Marcos Bedo ${ }^{1}$ \\ ${ }^{1}$ Fluminense Northwest Institute - Fluminense Federal University (UFF), Brazil \\ \{mnocelle, rodolfooliveira, gustavosemaan, marcosbedo\}eid.uff.br \\ ${ }^{2}$ Inst. of Comp. and Mathematics - Federal University of Itajubá (UNIFEI), Brazil \\ olmes@unifei.edu.br \\ ${ }^{3}$ Institute of Computing - Fluminense Federal University (UFF), Brazil \\ danielcmodic.uff.br \\ ${ }^{4}$ Federal Institute of North of Minas Gerais (IFNMG), Brazil \\ lucio.santoseifnmg.edu.br
}

\begin{abstract}
Decision-support systems benefit from hidden patterns extracted from digital information. In the specific domain of gastropod characterization, morphometrical measurements support biologists in the identification of land snail specimens. Although snails can be easily identified by their excretory and reproductive systems, the after-death mollusk body is commonly inaccessible because of either soft material deterioration or fossilization. This study aims at characterizing Brazilian land snails by morphometrical data features manually taken from the shells. In particular, we examined a dataset of shells by using different learning models that labeled snail specimens with a precision up to $97.5 \%$ $(F 1$-Score $=.975, C K C=.967$ and ROC Area $=.998)$. The extracted patterns describe similarities and trends among land snail species and indicates possible outliers physiologies due to climate traits and breeding. Finally, we show some morphometrical characteristics dominate others according to different feature selection biases. Those data-based patterns can be applied to fast land snail identification whenever their bodies are unavailable, as in the recurrent cases of lost shells in nature or private and museum collections.
\end{abstract}

\section{Introduction}

While land snails are a recurrent presence within the Brazilian tropical fauna scene, their identification and labeling are still a challenge since snail bodies quickly deteriorate after death. On the other hand, the shells, which are made of calcium carbonate, remain preserved long after. Accordingly, land snail shells are easily found in the wild and museums, and their investigation is one of the main sources in the characterization of such a class of gastropods [Ueta 1980, Slapcinsky and Kraus 2016]. For instance, chemical analyses enable the extraction of climate, food, and genomics information, usually destroying (parts of) the shell in the process [Simone 2006, Hirano et al. 2018].

A classical, non-invasive approach to label those shells is to $(i)$ portray their physiology and then (ii) compare the collected measures against a golden pattern or reference [Simone 2006, Queiroz 2007, Slapcinsky and Kraus 2016]. This 

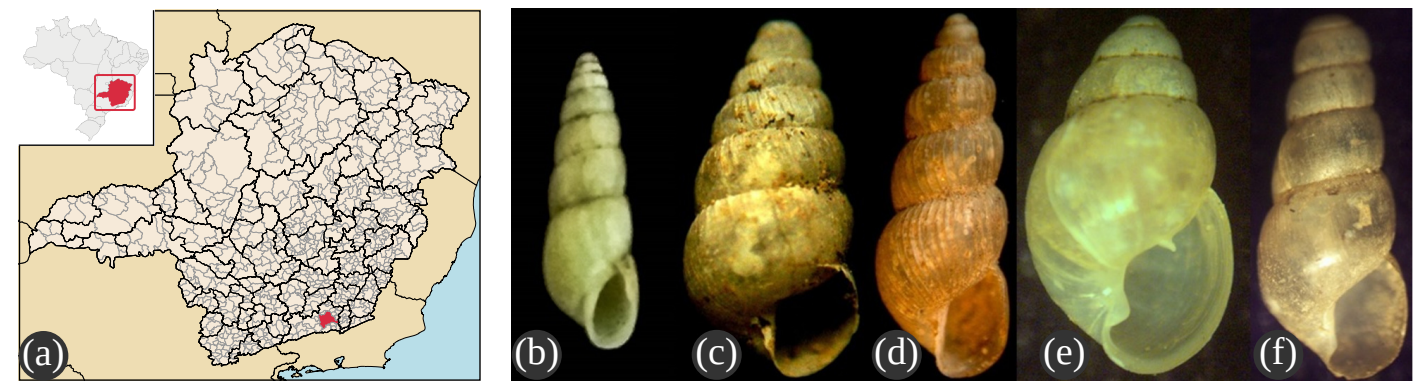

Figure 1. Brazilian land snails specimens. (a) Shells found at the Juiz de Fora/MG metro area. (b) Allopeas gracilis. (c) Beckianum beckianum. (d) Dysopeas muibum. (e) Leptinaria unilamellata. (f) Subulina octona.

morphometrical comparison process enables the fast identification of shells within collections and provides a rationale to find possible related species by similarity [Quenu and et. al 2020, Yeung and et. al 2020]. In this study, we take a step further to enhance such a comparison-based process by extracting data-driven patterns from different learning models tested over a handcrafted dataset of Brazilian land snail shells.

In particular, we curated a dataset (coined HELIX) ${ }^{1}$ that contains shells gathered at the metro area of the Brazilian city of Juiz de Fora/MG, whose land snail species are vastly documented ${ }^{2}$. Figure 1a shows the geographical location of shells, while Figure $1 \mathrm{~b}$ presents a representative shell instance for each of the five different HELIX species. Every shell was manually collected throughout a year, and the dataset covers a wide variety of individual mollusks of different genders and ages. Accordingly, we took full advantage of the KDD pipeline for mining HELIX data [Aggarwal 2015].

Pre-processed instances were feed to fine-tuned Naïve-Bayes, JRip, Decision Tree, RandomForest, Instance-based Learning, Functional-Tree, and Multi-Layer Perceptron (MLP) classifiers, which excelled in the labeling task with a precision up to $97.5 \%$. Accordingly, we examined the learned models and found patterns showing $(i)$ a geometric separation between the specimens, (ii) similar land snails according to their shell morphometrics, (iii) correlations between hierarchical characteristics and the snail species, (iv) rules for associating a subset of shell features to the species, and $(v)$ the dominance of certain characteristics in the labeling processes. Such data-driven outcomes provide insights to the comparison process by explicitly describing the morphometrical patterns of land snail shells as well as highlighting spatial similarity trends.

The remainder of this paper is organized as follows. Section 2 provides the background and related work, while Section 3 describes the material and methods. Finally, Sections 4 and 5 provide the experimental evaluation and conclude the study.

\section{Preliminaries and Related Work}

The morphometrical comparison process of land snails relies on portraying and measuring the snail shells for further matching against baselines of independent and correlated morphological characteristics. For instance, the study of Ueta (1980) provides a morphometrical catalog for Brazilian land snails. The comparison process can also be coupled

\footnotetext{
${ }^{1}$ HELIX dataset is available at zenodo.org/record/5500215\#.YUCgD $51 \mathrm{Kg} 2 \mathrm{w}$

${ }^{2}$ See ufje.br/malacologia
} 


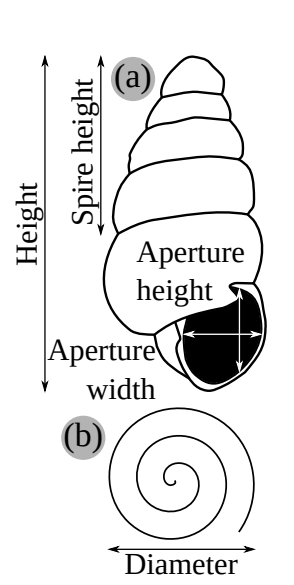

\begin{tabular}{l|l}
\hline \hline Rule $\downarrow$ (All measures are in mm.) & Species \\
\hline Aperture height $\leq 2.1 \wedge$ Diameter $\leq 2.1$ & D. muibum \\
\hline Aperture height $>2.1 \wedge$ Diameter $>4.7$ & L. unilamel. \\
\hline Aperture height $\leq 2.1 \wedge 2.1<$ Diameter $\leq 2.4 \wedge$ Height $\leq 4.8$ & B. beckianum \\
\hline Aperture height $\leq 2.1 \wedge 2.1<$ Diameter $\leq 2.4 \wedge$ Height $>4.8$ & S. octona \\
\hline Aperture height $\leq 2.1 \wedge$ Diameter $>2.4$ & B. beckianum \\
\hline $2.1<$ Aperture height $\leq 2.9 \wedge$ Diameter $\leq 4.7$ & S. octona \\
\hline Aperture height $>2.9 \wedge$ Diameter $\leq 4.7 \wedge$ Spire height $\leq 5.8$ & A. gracilis \\
\hline Ap. ht. $\leq 3.1 \wedge$ Diameter $\leq 3.6 \wedge$ Spire height $>5.8$ & S. octona \\
\hline $3.1<$ Ap. ht. $\leq 3.5 \wedge$ Dia. $\leq 3.6 \wedge$ S. ht. $>5.8 \wedge$ Ht. $\leq 11.2$ & A. gracilis \\
\hline $3.1<$ Ap. ht. $\leq 3.3 \wedge$ Dia. $\leq 3.6 \wedge$ S. ht. $>5.8 \wedge$ Ht. $>11.2$ & S. octona \\
\hline $3.3<$ Ap. ht. $\leq 3.5 \wedge$ Dia. $\leq 3.6 \wedge$ S. ht. $>5.8 \wedge$ Ht. $>11.2$ & A. gracilis \\
\hline $2.9<$ Ap. ht. $\leq 3.5 \wedge 3.6<$ Diameter $\leq 4.7 \wedge$ S. ht. $>5.8$ & S. octona \\
\hline Ap. ht. $>3.5 \wedge$ Diameter $\leq 4.7 \wedge 5.8<$ Spire height $\leq 9.9$ & A. gracilis \\
\hline Ap. ht. $>3.5 \wedge$ Diameter $\leq 4.7 \wedge$ Spire height $>9.9$ & S. octona \\
\hline \hline
\end{tabular}

Figure 2. Schema for (a) vertical and (b) horizontal morphometrical shell features, and generative rules (induced by Figure 4) for the HELIX dataset.

with genomics to $(i)$ expand current taxonomies and (ii) identify relationships between extant and extinct species [Queiroz 2007]. For instance, the study of Hirano et. al (2018) uses morphometric traits combined with phylogenetic relationships to demonstrate an ocean snail considered extinct was still extant. Yeung et. al (2020) also relied on morphometrics to introduce a new Hawaiian snail that was not related to any known local species. Analogously, the study of Slapcinsky and Kraus (2016) uses a morphometrical comparison to review the existing Palaus species and identify new morphological characteristics.

Recently, learning models have been used to enhance and speed up the comparison process, as in the study of Quenu et. al (2020). The authors employed two learning models for the identification of phenotypes from New Caledonia snail species: an MLP classifier and a Gaussian mixture model. The results indicated a proportion of the $1 / 3$ of individuals was in an overlapped cluster among the patterns of two species.

\section{Material and Methods}

HELIX dataset. The Subulinidae family of Brazilian land snails includes similar species with particular morphometrical characteristics. We surveyed the metro area of the Juiz de Fora/MG city and collected snails from a representative private property with $62,000 \mathrm{~m}^{2}$ centered at $21^{\circ} 42^{\prime} 31^{\prime \prime} S, 43^{\circ} 21^{\prime} 26^{\prime \prime} \mathrm{W}$, alt. $795 \mathrm{~m}$, with red-yellow oxisol, $8.5 \mathrm{pH}$, and Cwa climate. We performed monthly collections in a ecosystem with Pennisetum purpureum, Brachiaria mutica, Paspalum notatum, Bidens pilosa, Leucena leucocephala, and Ricinus communis plants near a river flow in the 2008/Sep - 2009/Aug timespan. There, we defined an equally spaced transect of $200 \mathrm{~m}$ with ten collection points and gathered $50 \times 50 \mathrm{~cm}, 500 \mathrm{~g}$ litter-falls. Samples were sieved by $2.0 \mathrm{~mm}$ meshs, and living specimens were cleaned and fixed in a Railliet-Henry liquid, their shells removed, dried, and separated. Finally, we followed the guidelines of Simone (2006) to label the species and create the HELIX dataset. We measured each shell Height, Diameter, Spire height, and Aperture width and height with a pachymeter. Figures $2 \mathrm{a}-\mathrm{b}$ show the representation of the characteristics. The dataset includes 518 instances labeled as B. beckianum (28.8\%), D. muibum (21.2\%), A. gracilis (9.7\%), L. unilamellata (12\%), and S. octona (28.3\%). 
Table 1. Feature selection with MedianRank aggregation.

\begin{tabular}{c|c|c}
\hline \hline Pearson $\downarrow$ & Relieff $\downarrow$ & FCBF $\downarrow$ \\
\hline \hline Aperture ht. $(.47)$ & Aperture ht. $(.23)$ & Diameter $(.78)$ \\
Height $(.42)$ & Height $(.17)$ & Height $(.58)$ \\
Spire ht. $(.39)$ & Aperture wt. $(.16)$ & Aperture ht. $(.53)$ \\
Aperture wt. $(.37)$ & Diameter $(.14)$ & Spire ht. $(.46)$ \\
Diameter $(.27)$ & Spire ht. $(.11)$ & Aperture wt. $(.45)$ \\
\hline \hline
\end{tabular}

Table 2. Consolidated best/worst results for all batches and setups.

\begin{tabular}{c|c|c|c|l}
\hline \hline Classifier $\downarrow$ & F1 & CKC & ROC & Parameters \\
\hline \hline Naïve-Bayes & $.870 / .792$ & $.827 / .768$ & $.981 / .941$ & Normal, non-conditional d.p.f. \\
\hline JRip & $.936 / .859$ & $.917 / .818$ & $.982 / .947$ & 03 folds rule-finding \\
\hline Decision-Tree & $.947 / .835$ & $.932 / .791$ & $.975 / .945$ & Pruned, n-ary tree w/ entropy \\
\hline RandomForest & $.952 / .864$ & $.939 / .823$ & $.996 / .967$ & 100 iterations, 30-inst. per bag \\
\hline IB Learning & $.965 / .850$ & $.955 / .806$ & $.986 / .945$ & 03 neighbors w/ $L_{2}$ distance \\
\hline Functional-Tree & $.968 / .855$ & $.959 / .817$ & $.994 / .961$ & Oblique tree w/ linear function \\
\hline \hline ML Perceptron & $.975 / .858$ & $.967 / .822$ & $.998 / .954$ & 01 hidden layer w/ 05 neurons \\
\hline \hline
\end{tabular}

Tools and learning models. We examined HELIX data by using seven classifiers of distinct paradigms implemented by the Weka workbench ${ }^{3}$ coded in Java and binded into R $3.6 .3 \mathrm{v}(2020-02-29)$ through package 'RWeka'4. We also implemented the intrinsic dimensionality estimator [Chávez et al. 2001] for PCA transformation and feature selection in R. Feature selection procedures were implemented according to the Pearson, Relieff, and Fast Correlation-Based Filter (FCFB) criteria [Roffo 2017] with individual rankings aggregated with the MedianRank algorithm [Fagin et al. 2003]. As a result, we tuned the classifiers by using the original HELIX data and its versions with $(i)$ selected features and (ii) PCA-transformed instances. Each of the three data versions was scaled and feed in different and isolated batches to the models following a 10-fold cross-validation routine. We experimented with a broad set of parameters aiming to avoid overfitting and repeated the execution of randomized approaches $10 \times$ with distinct seeds.

\section{Experiments}

We constructed three test batches representing the $(i)$ original instances, $(i i)$ instances with selected features, and (iii) PCA-transformed data by using the rounded HELIX intrinsic dimension (equals 2). Feature selection was carried out with three filters and aggregated with MedianRank algorithm, as described in Table 1. The characteristics Aperture height and Height were the two most dominant and, consequently, were used to construct the second batch of HELIX tests. Table 2 shows the best and worst performances achieved by the classifiers and their tuning parameters for each batch test. Results indicate the labeling-driven learning models are suitable to the shell identification problem, as their performances were bounded into the $[.792, .975] \mathrm{F} 1-$ Score interval. Accordingly, we examined every learned model to describe the patterns and their biological interpretations.

Distance-based relationships and boundaries. The PCA visualization in Figures $3 \mathrm{a}-\mathrm{b}$ show L. unilamellata and D. muibum are spatially separated and clustered in Euclidean-

\footnotetext{
${ }^{3}$ Available at: cs.waikato.ac.nz/ml/weka/

${ }^{4}$ Available at: cran.r-project.org/web/packages/RWeka/index.html
} 


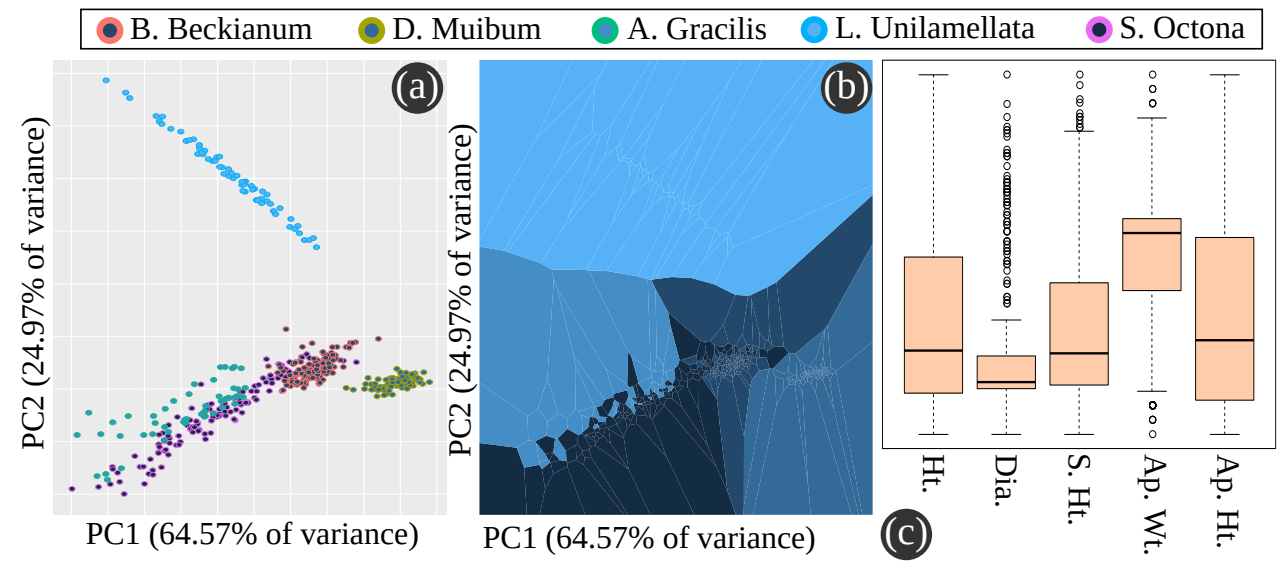

Figure 3. (a) PCA data visualization, (b) Voronoi-based similarity coverage for each land snail, and (c) $[0,1]$-scaled box-plot of HELIX attributes.

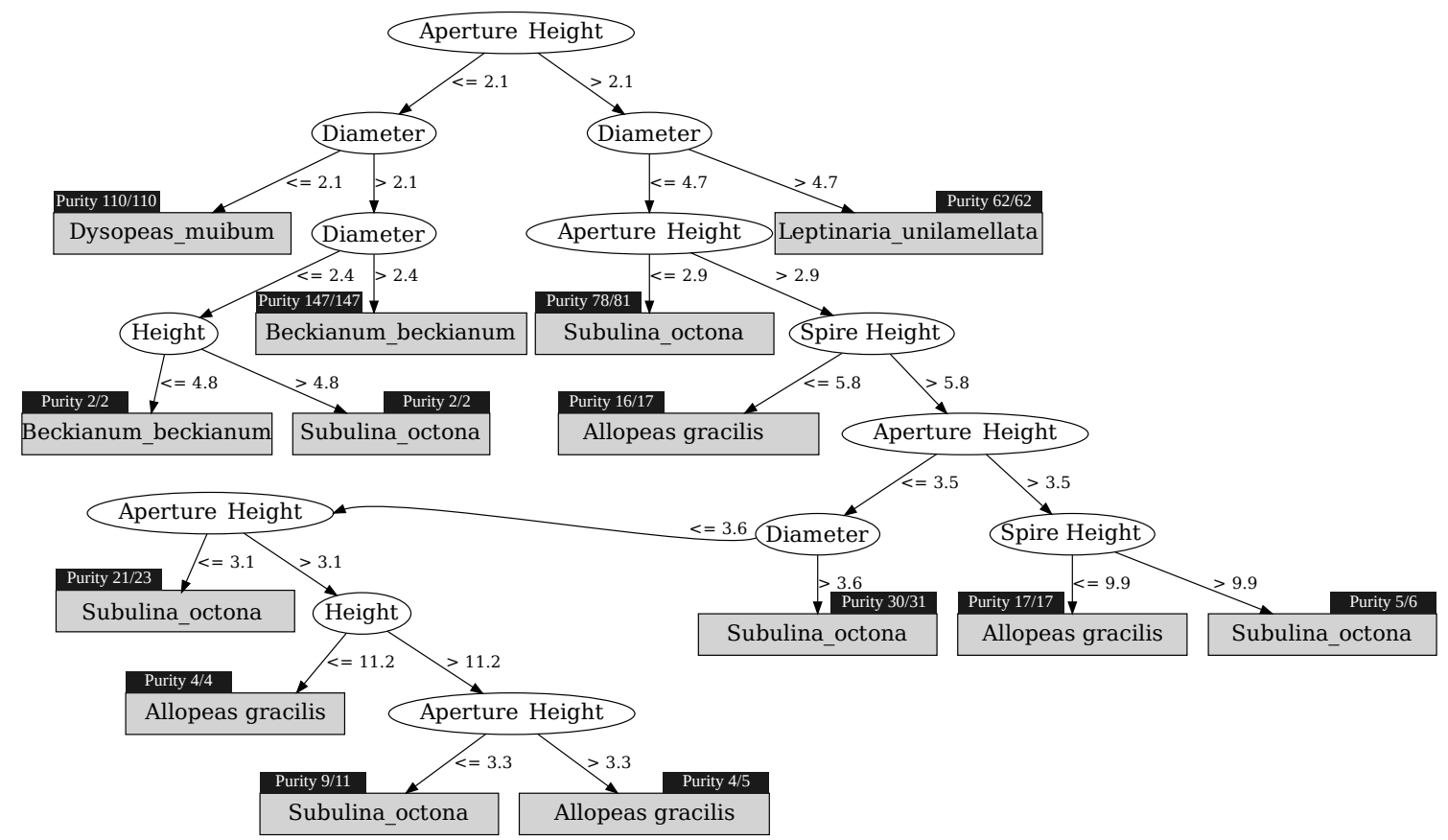

Figure 4. Decision-Tree constructed by the $\mathrm{C} 4.5$ learning model.

based Voronoi spaces with soft borders, which is biologically explained by morphometrics, i.e., the former land snails have bulkier shells and the latter shortened ones. The results also indicate two cluster overlappings among B. beckianum, S. octona and A. gracilis specimens, which is explained by the higher phylogenetical proximity among those species. Neither of those observations can be made only by checking individual nor pairwise attributes - Figure 3c.

Decision-Tree and association rules. The most frequent decision-tree constructed after the cross-validation procedure in Table 2 is presented in Figure 4. It describes $(i)$ a hierarchical correlation of attributes with shorter paths for describing L. unilamellata and D. muibum (corroborating Figure 3b), and (ii) a generative rule set that is detailed in Figure 2. The decision root node is the Aperture height (matching Table 1), and the tree paths show the most internal species is the A. gracilis, which can only be reached if at 
least three attributes are determined. Results also show the densest and pure nodes are in the first two levels, indicating Height and Diameter are used to separated species' inner borders. Such findings are biologically interpreted as B. beckianum, S. octona and A. gracilis specimens having similar Aperture height and Diameter depending on their age and eco-climate traits. Finally, generative rules constructed after the Decision-Tree have shown support of at least $80 \%$, which indicates those data-based patterns can be applied for fast land snail identification whenever their bodies are unavailable, as in the recurrent cases of lost shells in nature, private, or museum collections.

\section{Conclusions and Future Work}

This study has discussed a data-driven characterization of Brazilian land snails. In particular, we have $(i)$ curated a reference dataset by manually gathering and measuring a set of land snail shells at the metro area of the Brazilian city of Juiz de Fora/MG and (ii) evaluated those data by different learning models. Results indicate classifier models labeled land snail shells with a precision up to $97.5 \%$ (F1-Score $=.975, \mathrm{CKC}=.967$, and ROC Area $=.998)$, whereas learned models presented patterns regarding the proximity of species and the generative rules for the specimens. Such outcomes provide insights into the land morphometrical comparison process by describing data-driven trends. Future works include the HELIX extension to include other Brazilian land snail species.

Acknowledgments. The study was supported by CNPq, CAPES, and FAPERJ.

\section{References}

Aggarwal, C. (2015). Data mining: The textbook. Springer.

Chávez, E., Navarro, G., Baeza-Yates, R., and Marroquín, J. (2001). Searching in metric spaces. In Computing Surveys, volume 33, pages 273-321. ACM.

Fagin, R., Kumar, R., and Sivakumar, D. (2003). Efficient similarity search and classification via rank aggregation. In ACM SIGMOD, pages 301-312.

Hirano, T., Wada, S., Mori, H., Uchida, S., Saito, T., and Chiba, S. (2018). Genetic and morphometric rediscovery of an extinct land snail on oceanic islands. J. of Molluscan S., 84(2):148-156.

Queiroz, K. (2007). Species concepts and species delimitation. Sys. Bio., 56(6):879-886.

Quenu, M. and et. al (2020). Geometric morphometrics and ML challenge currently accepted species limits of the land snail Placostylus. J. M. Studies, 86(1):35-41.

Roffo, G. (2017). Ranking to learn and learning to rank: On the role of ranking in pattern recognition applications. arXiv preprint arXiv:1706.05933.

Simone, L. R. L. d. (2006). Land and freshwater molluscs of Brazil. Museu de Zoologia, Universidade de São Paulo.

Slapcinsky, J. and Kraus, F. (2016). Revision of partulidae of Palau, with description of a new genus for an unusual ground-dwelling species. ZooKeys, 86(614):27.

Ueta, M. T. (1980). Estudo morfométrico da concha de Lymnaea columella. R. Soc. Bras. Medicina Tropical, 13(1):119-141.

Yeung, N. and et. al (2020). Overlooked but not forgotten: the first new extant species of hawaiian land snail described in 60 years, Auriculella gagneorum. ZooKeys, 950:1. 